\title{
A proposed relationship between the unidimensional short form of the TMAS and the DAS: The effects of embedding vs. separate administration
}

\author{
JAMES L. TRAMILL \\ Department of Instructional Services, Wichita State University, Wichita, Kansas 67208 \\ and \\ STEPHEN F. DAVIS, SARAH BREMER, MICHAEL M. DUDECK, \\ and DAVID L. ELSBURY \\ Emporia State University, Emporia, Kansas 66801
}

\begin{abstract}
The relationship between the Death Anxiety Scale (DAS) and the unidimensional short form of the Taylor Manifest Anxiety Scale (TMAS) was studied. A second purpose of the study was to evaluate the effects of administering a combined, or embedded, version of these two instruments. These tests were administered to 236 undergraduate college students. Regardless of type of administration, the DAS and short form of the TMAS were highly and positively related. Further, females scored significantly higher than males on both instruments. Administration of the combined version resulted in a decrease, albeit nonsignificant, in the scores on both tests.
\end{abstract}

Judging from its reported usage, one of the most popular instruments currently being employed to evaluate fear of death or death anxiety is the Death Anxiety Scale (DAS), a 15-item true-false questionnaire, developed by Templer (1970). Representative data gathered through the use of the DAS have included the assessment of death anxiety in a variety of collegestudent groups, psychiatric patients, male and female apartment house residents, male and female adolescents, male suicide attempters (Templer \& Ruff, 1971), inpatient alcoholics (Sillman, 1981), and career military officers and their wives (Koob \& Davis, 1977). Templer (1970) reported that the validation procedures used for the DAS included comparisons of DAS scores of clinically diagnosed high-death-anxiety patients with those of a control group, correlation of DAS scores with scores from Boyar's (1964) Fear of Death Scale (FODS), and the correlation of DAS scores with the Welsh Anxiety Scale, the Taylor Manifest Anxiety Scale (TMAS), and subtests of the Minnesota Multiphasic Personality Inventory (MMPI). Such procedures have clearly shown that patients diagnosed as having high anxiety had significantly higher DAS scores, and the DAS and FODS were positively and significantly correlated with both the Welsh Anxiety Scale and the TMAS. However, as the correlation between the DAS and the Welsh Anxiety Scale $(r=.39)$ and the correlation between the DAS and the TMAS $(r=.36)$ were lower than the correlation of the TMAS with the Welsh Anxiety Scale $(r=.78)$, it was felt (Templer, 1970) that the DAS was measuring something other than general anxiety, that is, that the DAS possessed what Campbell and Fiske (1956) have termed discriminant validity.

The positive correlation between the DAS and the TMAS notwithstanding, several factor-analytic studies (Kahn, 1970; Notz, 1972; O'Connor, Lorr, \& Stafford, 1956) have indicated that the TMAS may, in fact, measure a composite of several factors. In an attempt to rectify this situation, Hicks, Ostle, and Pellegrini (1980) have developed a unidimensional short form of the TMAS. The intended purpose of this short form was to measure one dimension, general anxiety. Hicks et al. (1980) reported high test-retest and internal consistency correlations (.88 and .92 , respectively). On the other hand, further validation studies for this new instrument have, to the best of our knowledge, not appeared in the literature.

Given this pattern of results with regard to the development of the short form of the TMAS and the already established relationship between the DAS and the long form of the TMAS, it would appear to be of interest to ascertain the nature of the relationship that exists between the DAS and the short form of the TMAS. Should a modest correlation, similar to those previously reported, be found, it would appear to be supportive of the discriminant validity of the DAS. However, should a substantially higher correlation be reported, the question of the relationship between these 
two instruments and exactly what they measure would need further consideration and reassessment.

As the process of embedding, or combining, tests to form one composite instrument is frequently encoun tered in the psychological literature (the MMPI would appear to be an excellent example), a second purpose of the present study was to determine the effects of administering a combined version of the DAS and the shortform TMAS.

\section{METHOD}

\section{Subjects}

A total of 236 undergraduate college students $(95$ males, 141 females) served as subjects. All subjects were volunteers recruited from introductory psychology classes.

\section{Testing Instruments}

Three separate testing instruments were employed: (1) the DAS only, (2) the short-form TMAS only, and (3) a combination of these two formed by simply alternating questions from these instruments.

\section{Procedure}

Testing was done on two separate occasions, with all tests administered in the regular classroom setting. The second testing session took place exactly 4 weeks after the first administration. All possible orders of presentation and test combination were employed. For example, some subjects received the DAS only at the first test session and the DAS-TMAS combination at the second session, whereas the converse held true for other subjects, and so forth, until all possible sequences were accounted for.

\section{RESULTS AND DISCUSSION}

Generally speaking, a high, positive relationship was found to exist between the DAS and short-form TMAS. More specifically, the correlation between these two tests when they were administered independently to the same subjects at the two testing sessions was .73. The correlation of these two instruments when the scores were obtained from administration of the combined version was .77 . In addition to indicating that these two instruments are highly and positively related, regardless of type of administration, it should be noted that these correlations are considerably higher than those originally reported (Templer, 1970) between the long form of the TMAS and the DAS. On one hand, these results could be seen as being supportive of the Hicks et al. (1980) efforts to produce a unidimensional testing instrument; that is, the removal of other, unrelated, factors resulted in an elevation of the correlation. However, these higher correlations do raise the question of exactly what is measured by these two instruments. At least two possibilities would appear to exist. For example, the instruments might simply measure general anxiety, or they might both measure death anxiety. Unfortunately, the present data are not able to distinguish between these two possibilities. A third possibility might also be entertained. In neither case did either correlation account for more than $60 \%\left(\mathrm{r}^{2}\right)$ of the variance. Thus, other unaccounted variables are certainly present. Given the previous validation studies of the DAS with the FODS and high-death-anxiety patients and the accumulation of data reflecting differences in DAS scores among various occupations, one of these unaccounted factors might well be fear of death or death anxiety. This interpretation points to a more clearly defined path for future research in this area.

Other aspects of the present data also warrant consideration. First, corroborating several previously reported studies (e.g., Koob \& Davis, 1977; Sillman, 1981; Templer, Lester, \& Ruff, 1974; Templer \& Ruff, 1971), DAS scores (regardless of separate or embedded administration) of female subjects (first administration, mean $=7.01$; second administration, mean $=6.49$ ) were significantly higher than those of male subjects (first administration, mean $=6.36$; second administration, mean $=5.72$ ) [first administration, $F(1,165)=4.79$, $p<.05$; second administration, $F(1,167)=5.21, p<.05]$. The same pattern of results held true for the TMAS. Female subjects (first administration, mean $=8.66$; second administration, mean $=8.27$ ) scored significantly higher than did male subjects (first administration, mean $=7.59 ;$ second administration, mean $=7.18$ ) [first administration, $\quad F(1,174)=5.39, \quad p<.05 ; \quad$ second administration, $\mathrm{F}(1,186)=5.61, \mathrm{p}<.05]$.

Second, as reflected by these means, the general effect of administering these two instruments via the combined, or embedded, form was to reduce scores on both tests relative to scores obtained by separate administration. Even though such reductions failed to reach significance when compared with scores obtained under separate administrations, they were observed in literally all instances. This trend, also nonsignificant, was previously reported by Templer and Ruff (1971), who found that DAS items, when embedded in the MMPI, were depressed relative to scores on nonembedded items. This pattern of results suggests that caution should be exercised if one is considering the simultaneous use of these two, or similar, instruments.

\section{REFERENCES}

Boyer, J. I. The construction and partial validation of a scale for the measurement of the fear of death. Unpublished doctoral dissertation, University of Rochester, 1964.

Campbell, D. T., \& Fiske, D. W. Convergent and discriminant validation by the multitrait-multimethod matrix. Psychological Bulletin. 1956, 59, 81-105.

Hicks, R. A., Ostre, J. R., \& Pellegrini, R. J. A unidimensional short form of the TMAS. Bulletin of the Psychonomic Society, 1980, 16, 447-448.

KaHN, S. B. Dimensions of manifest anxiety and their relationship to college achievement. Journal of Consulting and Clinical Psychology, 1970, 35, 223-228.

Kоов, P. B., \& DAvis, S. F. Fear of death in military officers and their wives. Psychological Reports, 1977, 40, 261-262.

Notz, V. R. The factor structure of tests of anxiety. Unpublished master's thesis, San Jose State University, 1972.

O'Connor, J. P., Lorr, M., \& STAFFord, J. W. Some patterns of 
manifest anxiety. Journal of Clinical Psychology, 1956, 12, 160-165.

Sillman, C. L. Death anxiety, self-esteem, and locus of control in alcoholics. Unpublished master's thesis, Emporia State University, 1981.

TEMPLER, D. I. The construction and validation of a death anxiety scale. Journal of General Psychology, 1970, 82, 165-177.
Templer, D. I., Lester, D., \& RufF, C. F. Fear of death and feminity. Psychological Reports, 1974, 35, 530.

Temple R, D. I., \& RufF, C. F. Death anxiety scale means, standard deviations, and embedding. Psychological Reports, 1971, 29, 173-174.

(Received for publication January 16, 1982.) 